\title{
Manual de cuidados de enfermagem para pacientes pré e pós-operatório de colecistectomia: elaboração e avaliação
}

Nursing care manual for pre and post-operative cholecystectomy patients: elaboration and evaluation

Manual de cuidado de enfermería para pacientes pre y postoperatorios de colecistectomía: elaboración y evaluación

\begin{abstract}
Resumo
Objetivo: elaborar e avaliar um manual de cuidados de enfermagem para pacientes em pré e pós-operatório de colecistectomia. Metodologia: estudo elaborado a partir de um projeto de desenvolvimento que seguiu oito etapas metodológicas e a avaliação foi realizada por 48 participantes, entre eles docentes, enfermeiros, estudantes, pacientes e familiares, numa universidade federal e num hospital universitário do sul do Brasil. A análise foi por meio de frequências simples e absolutas para respostas fechadas e para as respostas abertas utilizou-se a Análise Textual Discursiva. Resultados: de forma unânime, os participantes acreditam que as informações contidas no manual são importantes, além de que a forma de disposição das informações é adequada e as ilustrações contribuem para o melhor entendimento do texto. Conclusão: a utilização do manual é válida quando incentiva para a educação em saúde; mostra os cuidados/procedimentos a serem realizados e proporciona bem-estar.
\end{abstract}

Palavras-chave: Educação em saúde; Manuais; Colecistectomia; Enfermagem perioperatória; Cuidados de enfermagem.

\begin{abstract}
Objective: to develop and evaluate a nursing care manual for patients in the pre- and postoperative period of cholecystectomy. Methodology: study based on a development project that followed eight methodological steps and the evaluation was carried out by 48 participants, including professors, nurses, students, patients and family members, at a federal university and a university hospital in southern Brazil. The analysis was through simple and absolute frequencies for closed answers and for open answers we used the Discursive Textual Analysis. Results: unanimously, the participants believe that the information contained in the manual is important, in addition to the fact that the way the information is arranged is adequate and the illustrations contribute to a better understanding of the text. Conclusion: the use of the manual is valid when it encourages health education; shows the care/procedures to be performed and provides well-being.
\end{abstract}

Keywords: Health education; Manuals; Cholecystectomy; Perioperative nursing; Nursing care. 


\begin{abstract}
Resumen
Objetivo: desarrollar y evaluar un manual de cuidados de enfermería para pacientes en el pre y postoperatorio de colecistectomía. Metodología: estudio basado en un proyecto de desarrollo que siguió ocho pasos metodológicos y la evaluación fue realizada por 48 participantes, entre profesores, enfermeras, estudiantes, pacientes y familiares, en una universidad federal y un hospital universitario en el sur de Brasil. El análisis fue a través de frecuencias simples y absolutas para respuestas cerradas y para respuestas abiertas usamos el Análisis Textual Discursivo. Resultados: por unanimidad, los participantes creen que la información contenida en el manual es importante, además de que la forma en que se ordena la información es adecuada y las ilustraciones contribuyen a una mejor comprensión del texto. Conclusión: el uso del manual es válido cuando fomenta la educación para la salud; muestra los cuidados/procedimientos a realizar y proporciona bienestar.
\end{abstract}

Palabras clave: Educación para la salud; Manuales; Colecistectomía; Enfermería perioperatória; Cuidado de enfermera.

\title{
1. Introdução
}

A remoção cirúrgica da vesícula biliar (colecistectomia) tem como objetivo a solução das doenças vesiculares. Pode ser realizada mediante técnicas cirúrgicas tradicionais como a colecistectomia convencional ou pelo método de laparoscopia, realizada através de uma pequena incisão na cicatriz umbilical, sendo considerada a mais utilizada na atualidade (Hinkle \& Cheever, 2015).

Segundo dados internacionais, estima-se que anualmente nos Estados Unidos cerca de 750.000 pacientes são submetidos à colecistectomia (Stinton \& Shaffer, 2012). No contexto brasileiro, dentro do âmbito do Sistema Único de Saúde (SUS), no período de 2016 o número total de procedimentos de colecistectomia foi de 194.570, desses 122.209 realizados mediante a técnica convencional e 72.361 pelo método de laparoscopia (Ministério da Saúde, 2016). Pode ser estimado que cerca de 540 procedimentos de colecistectomia (convencional e laparoscópica) são realizados por dia no Brasil.

Tendo em vista a importância de uma assistência de qualidade para pacientes que serão submetidos a um procedimento cirúrgico, é essencial que enfermeiros em conjunto com a equipe multiprofissional forneça um atendimento que envolva as necessidades do paciente ao longo desse período, permeando cuidados que englobem não somente aspectos físicos, mas espirituais e psicológicos. O estabelecimento de uma relação de confiança entre enfermeiro-paciente-familiar pode facilitar a assistência e gerar benefícios como a minimização de ansiedade, angústia, medo e dúvidas (Benevides, Silva, Silva \& Farias, 2020).

Destaca-se como uma das responsabilidades da equipe enfermagem, participar integralmente durante o cuidado do paciente cirúrgico, com o objetivo de proporcionar bem-estar a esse paciente e a sua família. Acredita-se que uma comunicação eficaz é fundamental para o estabelecimento de uma relação terapêutica entre enfermeiro-paciente/família, tornando-se possível a execução de uma assistência qualificada, integral e individualizada (Ascari et al., 2013). Desse modo, o enfermeiro deve proporcionar para o paciente e sua família a compreensão do processo cirúrgico que será submetido.

As orientações de enfermagem devem ser explanadas de maneira clara e objetiva, com o intuito de minimizar dúvidas e receios em relação a situação vivenciada. Assim, torna-se possível interferir diretamente no processo de recuperação do paciente, na redução de riscos cirúrgicos, como também no tempo de internação hospitalar, além de oportunizar a diminuição de complicações pós-operatórias por meio do fornecimento de cuidados qualificados no período pré-operatório (Costa, Dias, Azevedo \& Silva, 2015).

Neste contexto, o enfermeiro deve pensar a prática educativa como inerente aos cuidados de enfermagem, numa perspectiva de ação-reflexão-ação dialógica e conscientizadora. Como educador, o profissional auxilia na transformação, autonomia e emancipação dos indivíduos. A prática educativa vem de encontro ao aumento da visualização e da valorização da profissão, que consequentemente refletirá maior reconhecimento e ampliação do espaço de atuação profissional (Rigon \& Neves, 2011). 
A unificação dos cuidados de enfermagem com o uso de materiais educativos é essencial para a promoção de uma assistência de qualidade. A ação de educação do paciente reflete significativamente na melhora do seu conhecimento e satisfação, na diminuição da ansiedade, na evolução do sentimento de segurança, apoio emocional e confiança, propiciando melhor qualidade de vida para o paciente e seus familiares (Gurusamy, Vaughan \& Davidson, 2014).

A família dos pacientes internados também deve ser considerada pelo enfermeiro e estar inclusa nas estratégias de educação em saúde. Esses, acompanham quase que em turno integral o paciente no ambiente hospitalar e na maioria das vezes exercem a função de cuidador domiciliar pós-alta. Dessa maneira, torna-se relevante que entendam os cuidados específicos e as orientações que devem ser seguidas para a diminuição do risco de complicações através do reconhecimento de alterações primárias (Silva, Monteiro \& Santos, 2015).

Dentre os diversos recursos para comunicação, encontram-se os materiais ilustrativos que facilitam a compreensão e a fixação de informações, são estratégias precisas e práticas para educar (Mafetoni, Higa \& Bellini, 2011). Manuais de orientações apresentam-se como instrumentos contribuintes para enfermeiros no âmbito da educação em saúde. Esses, são capazes de nortear ações de cuidados em diferentes cenários, contribuindo para o desenvolvimento da capacidade de interferência dos pacientes e familiares nos processos decisórios, o que reflete no crescimento da autonomia e prevenção de complicações de saúde (Nascimento et al., 2015).

Nessa perspectiva e considerando o elevado índice de procedimentos de colecistectomia no âmbito nacional e internacional, detecta-se ser imprescindível desenvolver manuais de orientações e cuidados de enfermagem que abranjam esses pacientes. Tendo em vista ainda, os benefícios que um manual oferece e que dispor de tecnologia para a educação facilita e uniformiza as orientações da equipe de saúde, contribuindo inclusive com a prática clínica dos enfermeiros, além de proporcionar bem-estar físico, mental e social para os pacientes e familiares. Assim, o estudo objetivou elaborar um manual de cuidados de enfermagem para pacientes pré e pós-operatório de colecistectomia e avaliar sua face e conteúdo.

\section{Metodologia}

Trata-se de um estudo elaborado a partir de um projeto de desenvolvimento realizado na Escola de Enfermagem (EEnf) da Universidade Federal do Rio Grande (FURG) e na Unidade de Clínica Cirúrgica (UCC) do Hospital Universitário Dr. Miguel Riet Corrêa Júnior (HU) filial da Empresa Brasileira de Serviços Hospitalares (EBSERH).

Para a elaboração do manual foram seguidas etapas metodológicas sobre construção de manuais de orientações e cuidados em saúde (Oliveira, Lucena \& Echer, 2014), sendo essas: elaboração de um projeto de desenvolvimento; definição dos conteúdos e fundamentação teórica; adaptação da linguagem e seleção de conteúdo; inclusão de ilustrações; finalização do manual piloto; avaliação do manual; layout do manual; impressão final e distribuição/acesso.

A avaliação de face e conteúdo do manual foi realizada por 48 participantes, distribuídos: todos os docentes da EEnf com experiência em cuidados de enfermagem na área cirúrgica $(n=4)$; todos os acadêmicos do oitavo semestre do curso de Graduação em Enfermagem da FURG, aprovados na disciplina de Enfermagem Perioperatória (n=5); todos os residentes da Residência Integrada Multiprofissional Hospitalar com ênfase na atenção à saúde cardiometabólica do adulto (RIMHAS) que atuaram na UCC ( $n=3)$; todos os enfermeiros assistenciais efetivos da UCC dos turnos tarde, noite 1 e noite 2 ( $n=3$ ); todos os pacientes em período pré ou pós-operatório de colecistectomia da UCC $(n=17)$ e seus familiares $(n=16)$ no mês de setembro de 2016. Justifica-se esse número devido ser o resultado de uma estimativa da média mensal do total de cirurgias de colecistectomia convencional e laparoscópica realizadas no ano de 2015 no HU-FURG-EBSERH. Identificando-se um total de 201 procedimentos e uma média mensal de 16,75 cirurgias (Hospital Universitário, 2016).

Os participantes foram selecionados de acordo com os critérios de inclusão, descritos: ser profissional com 
conhecimento na área cirúrgica, estudantes do curso de enfermagem aprovados na disciplina de Enfermagem Perioperatória, paciente em período pré ou pós-operatório de colecistectomia e seus familiares, ter condições cognitivas para ler e responder o manual e o instrumento, concordar em fazer parte do estudo e assinar o Termo de Consentimento Livre e Esclarecido (TCLE). Os critérios de exclusão estavam limitados à ausência dos participantes nos locais e no momento de coleta de dados.

A coleta de dados foi realizada no mês de setembro de 2016 por meio de dois encontros. No primeiro realizou-se esclarecimentos sobre o estudo junto com a entrega do TCLE, do manual piloto e do instrumento de avaliação, sendo esse um questionário autoaplicável, constituído por 10 questões abertas e fechadas relacionadas ao conteúdo/organização. O mesmo foi adaptado do instrumento de avaliação de manuais (Graciotto, Gomes, Sangalli, Schneider \& Echer, 2010). Ainda, foi possível a realização de contribuições escritas no próprio manual pelo participante através de sua disponibilização. Já o segundo encontro era agendado de acordo com a disponibilidade do participante e tinha como objetivo o recolhimento do material e agradecimento pela participação.

O procedimento de entrega dos documentos foi por intermédio de visitas à UCC e na EEnf, de maneira que os possíveis participantes foram convidados a participar no próprio local e turno de trabalho/estudo. Definiu-se um máximo de três tentativas aos diferentes locais para o recolhimento dos documentos previamente entregues. Para o acesso de pacientes em período pré e pós-operatório de colecistectomia, primeiramente foi realizada conversa prévia com a equipe de saúde da unidade, com o intuito de verificar seu estado clínico/cirúrgico e a sua possibilidade de participação.

A análise dos dados deu-se pela leitura do manual piloto que foi encaminhado para os participantes, juntamente com o instrumento de avaliação. Destaca-se que durante a análise do manual piloto, obteve-se atenção para sugestões, correções, inclusões e exclusões de conteúdo. As questões do instrumento de avaliação foram analisadas mediante frequências simples e absolutas das respostas fechadas, organizadas em planilha no programa Microsoft Office Excel do Windows. As respostas abertas das questões 1,9e 10 foram analisadas através da Análise Textual Discursiva (ATD) de Moraes \& Galiazzi (2014), já as respostas das demais questões abertas, foram utilizadas para modificações do próprio manual.

Os participantes da pesquisa foram identificados pela letra inicial das palavras correspondentes aos mesmos, seguidas de um algarismo. Exemplo: Docente 1 (D.1). Os aspectos éticos foram respeitados conforme as recomendações da Resolução 466/12 do Conselho Nacional de Saúde. O projeto foi submetido e aprovado sob o parecer 88/2016 pelo Comitê de Ética em Pesquisa na Área da Saúde (CEPAS) da FURG.

\section{Resultados}

De acordo com o primeiro objetivo proposto pelo estudo, foi elaborado o manual de cuidados de enfermagem, intitulado: Pré e pós-operatório de Colecistectomia: orientações e cuidados de enfermagem para pacientes e familiares. Para sua construção, foram seguidas oito etapas metodológicas (Oliveira, Lucena \& Echer, 2014).

Na primeira etapa realizou-se a construção de um projeto de desenvolvimento, o qual conteve introdução, objetivo, método, cronograma, orçamento, referências, TCLE e instrumento de avaliação do manual construído. Esse foi submetido e aprovado pelo CEPAS/FURG.

A segunda etapa visou definir os conteúdos que foram explanados no manual, buscou-se informações na literatura especializada para obter aprofundamento teórico no assunto de escolha. Na terceira etapa, os autores trabalharam na adaptação da linguagem, ou seja, a transformação do saber científico em informações que sejam de melhor compreensão para pacientes e familiares. É importante destacar que durante essa adaptação, foi possível evidenciar certa dificuldade na reescrita dessas informações. Nota-se que a conversão da linguagem é mais fácil quando realizada verbalmente, como por exemplo durante a 
prática de orientação. Ainda, nessa fase os conteúdos foram selecionados segundo sua relevância, evitando a sobrecarga do manual, conservando objetividade e atração para o usuário.

A quarta etapa caracterizou-se pela inclusão de ilustrações, com o intuito de facilitar o entendimento dos conteúdos, tornando o material mais atrativo, descontraído e menos pesado. Como obstáculo dessa etapa, aponta-se a dificuldade em encontrar imagens adequadas de acordo com as orientações que foram planejadas. Desse modo, emergiu a necessidade de criação de fotos, essas foram produzidas no laboratório de práticas da EEnf, Após a finalização do manual piloto, encaminhou-se o material para impressão, iniciando a quinta etapa: avaliação do manual.

A sexta etapa contemplou o layout do manual, visando tornar o material visivelmente atraente para o leitor. Nesse momento foram acertados os últimos detalhes, passando para a sétima etapa: impressão final. A oitava etapa compreendeu a distribuição/acesso.

O manual concretizou-se em 31 folhas sulfite tamanho A4. Quanto à impressão, realizou-se no formato frente e verso em orientação horizontal, cada folha foi dividida ao meio verticalmente, confeccionando-se cada página em ambas divisões em formato de livreto, sendo unidas através de grampeamento, totalizando neste formato 16 páginas. O material foi impresso em tinta colorida e foi utilizado recursos como caixas de textos e gravuras para destacar informações e chamar a atenção do leitor.

Em relação ao processo de avaliação, dentre todas as contribuições realizadas pelos participantes referentes às questões fechadas, destacam-se aquelas que apresentaram aspectos a serem modificados. Na questão que trata sobre as "informações estarem facilmente localizadas no manual", 10 (21\%) dos participantes opinaram que apenas na maioria das vezes foram localizadas com facilidade; na questão que relaciona o "tamanho e estilo da letra", seis $(12,5 \%)$ dos participantes referiram serem pouco adequados; na questão que aborda a "linguagem utilizada no material", três $(6,2 \%)$ participantes julgaram estar pouco acessível.

Em contrapartida, 48 (100\%) dos participantes acreditam que as informações contidas no manual são importantes, além de que a forma de disposição das informações é adequada e as ilustrações do manual contribuem para o melhor entendimento do texto.

Quanto às questões abertas do instrumento de avaliação, atentou-se para todas as sugestões de melhoria propostas pelos participantes, sendo utilizadas para modificações do próprio manual. Já as questões abertas 1,9 e 10 foram analisadas separadamente através da ATD, buscando o processo de categorização das informações.

A análise foi iniciada com a unitarização do texto, ou seja, a desmontagem por meio da fragmentação das respostas dos participantes, com o objetivo de obter as unidades de significado. O processo de unitarização originou frases, que neste caso foram consideradas as próprias unidades de significado, porém caso essas frases apresentassem mais de uma ideia, eram desdobradas e classificadas em duas ou mais unidades de significado. O passo seguinte, foi o agrupamento das unidades de significado semelhantes, constituindo-se as categorias iniciais.

Através da análise das frases foi possível organizar 10 categorias iniciais, apresentadas na Tabela 1 que apresenta os títulos dessas categorias e a distribuição quantitativa de unidades de significado (frases). 
Tabela 1 - Categorias iniciais organizadas a partir das unidades de significado.

\begin{tabular}{ccc}
\hline & Quantificação das categorias iniciais & Número de \\
\hline Categorias & Título da categoria & frases \\
Iniciais & Facilitador para orientações/conhecimentos & 11 \\
1 & Apresenta orientações importantes e necessárias & 5 \\
3 & Apresenta informações esclarecedoras & 12 \\
4 & Elucida os cuidados por meio das imagens & 3 \\
5 & Oferece empoderamento aos pacientes para o autocuidado & 5 \\
6 & Esclarece os cuidados/procedimentos a serem realizados & 7 \\
7 & Facilitador para cuidar dos pacientes & 2 \\
9 & Diminui o estresse/ansiedade dos pacientes e dos familiares & 5 \\
10 & Traz segurança e calma & 2 \\
& Contribui para a recuperação do paciente & 2
\end{tabular}

Fonte: Autores (2017).

Durante o processo de análise foi realizado o reagrupamento das categorias iniciais, promovendo reflexões acerca da avaliação do manual. No movimento de reagrupamento das categorias iniciais mais próximas, organizaram-se três categorias finais, sendo que no caso dessa análise não foi necessário a organização das categorias intermediárias. As categorias finais são apresentadas na Tabela 2 .

Tabela 2 - Categorias finais organizadas a partir das unidades de significado.

\begin{tabular}{|c|c|c|c|}
\hline \multirow[b]{2}{*}{ Categorias } & \multicolumn{3}{|c|}{ Quantificação das categorias finais } \\
\hline & Título da categoria & $\begin{array}{c}\text { Categorias iniciais ou sub- } \\
\text { categorias das categorias } \\
\text { finais }\end{array}$ & $\begin{array}{l}\text { Número de } \\
\text { frases }\end{array}$ \\
\hline I & Incentiva para a educação em saúde & $1,2,3$ & 28 \\
\hline II & $\begin{array}{c}\text { Mostra os cuidados/procedimentos a serem } \\
\text { realizados }\end{array}$ & $4,5,6,7$ & 17 \\
\hline \multirow[t]{2}{*}{ III } & Proporciona bem-estar & $8,9,10$ & 9 \\
\hline & Total & & 54 \\
\hline
\end{tabular}

Fonte: Autores (2017).

Pelos resultados analisados, os participantes do estudo percebem que o manual de cuidados de enfermagem para pacientes e familiares é válido visto que, incentiva para a educação em saúde; mostra os cuidados/procedimentos a serem realizados; e proporciona bem-estar.

As categorias finais encontradas por meio da ATD mostram os resultados da avaliação do manual, podendo assim ser compreendido os aspectos que o tornam pertinente para a utilização. 


\section{Discussão}

A partir do cumprimento da etapa de avaliação, podem ser discutidas questões que foram destacadas na apresentação dos resultados. Em relação à localização das informações no manual, alguns participantes apontaram que apenas na maioria das vezes as informações são facilmente localizadas. Sendo esse um critério de organização importante na construção de materiais educativos, pois é essencial que o leitor não sinta dificuldade em encontrar os conteúdos desejados, já que o objetivo maior do material é auxiliá-lo. Adequações com esse intuito foram realizadas na versão final do manual.

Destaca-se a necessidade de o layout ser visivelmente atraente. Para isso, é preciso dar atenção a apresentação do conteúdo, a partir de uma boa distribuição de texto, inclusão de gravuras/ilustrações e adequação do tamanho e estilo da letra (Oliveira, Lucena \& Echer, 2014).

Quanto ao quesito do tamanho e estilo da letra, apenas alguns acadêmicos, enfermeiros e residentes destacaram estarem pouco adequados. É interessante perceber que no ponto de vista dos pacientes e familiares em sua unanimidade a formatação estava adequada.

Esse resultado pode ser reflexo do comportamento de pacientes resignados ao que é ofertado e por parte dos acadêmicos (futuros profissionais) e profissionais uma preocupação com possíveis dificuldades visuais apresentadas pelos leitores. Segundo autores, pacientes possuem um perfil passivo e pouco questionador frente a prestações de cuidados, o que muitas vezes pode ser consequência do desconhecimento do seu direito às informações que deveriam ser fornecidas durante a internação hospitalar, como, também, de participar das decisões sobre seu cuidado e tratamento (Chaves, Costa \& Lunardi, 2005).

Em uma das etapas do processo de elaboração do manual objetivou-se a adaptação da linguagem, que quando disposta de maneira clara, estará colaborando para uma desconstrução de possíveis entraves no processo de comunicação, tornando o material mais eficiente. Assim, o emprego da linguagem científica dificulta o entendimento do leitor, logo o texto deve ser breve, direto e compreensível (Coriolano-Marinus, Pavan, Lima \& Bettencourt, 2014).

No que se refere a essa questão, 6,2\% dos participantes acreditam que a linguagem utilizada está pouco acessível. É importante atentar-se para esse dado, visto que um material educativo deve ser um fácil transmissor de informações e esclarecedor de dúvidas. É essencial evitar o efeito inverso da aplicabilidade do manual, ou seja, difusão de dúvidas em relação ao conteúdo apresentado.

Todos os participantes concordam que as informações contidas no manual são importantes e que as ilustrações contribuem para o melhor entendimento do texto. A primeira ideia, reflete-se na notável representatividade que o uso de manuais educativos na prática diária oferece. Nesse sentido, equivale-se a uma ferramenta que facilita, uniformiza e oficializa as condutas de cuidado, além de ser considerado um equipamento de suporte e reforçador das orientações verbais realizadas pelos profissionais de saúde, pode ser utilizado desde a internação até o cuidado domiciliar (Oliveira, Lucena \& Echer, 2014; Costa et al, 2018).

No que se diz respeito à inclusão de ilustrações, seu emprego é apontado como fator motivacional pra a leitura, além de atuar de forma complementar ao conteúdo escrito do material, contribui para o entendimento do assunto, devendo ser disposta de maneira a chamar a atenção dos usuários (Oliveira, Lopes \& Fernandes, 2014). Ainda, ajudam na fixação de conteúdo, facilitando a legibilidade e compreensão do texto (Oliveira, Lucena \& Echer, 2014), sendo essa condição necessária na produção de um material educativo de qualidade, eficiente e potente para alcançar pacientes e familiares.

Através da composição das categorias finais, foi possível compreender quando que os participantes percebem que a utilização do manual elaborado é válida. Sendo assim, quando incentiva para a educação em saúde, mostra os cuidados/procedimentos a serem realizados e proporciona bem-estar.

O fato do manual ser considerado como incentivador para a educação em saúde é primordial para o alcance do propósito 
principal dos materiais educativos. O manual em questão cumpre esse papel, a partir do momento em que é considerado um facilitador de orientações, apresentando orientações importantes e necessárias, além de informações esclarecedoras.

É preciso olhar para a prática de educar em saúde como uma ação que deve ser constante no trabalho dos enfermeiros e demais profissionais de saúde. Esses possuem papel crucial na educação, enquanto comprometidos com a saúde pública (Moura et al., 2017). Dentre as estratégias de educação, encontra-se a confecção de manuais de orientações, o qual é um veículo facilitador transmissor de informações. Neste contexto, o enfermeiro deve promover práticas educativas e utilizar de suas habilidades de educador como uma estratégia de cuidado permanente, de modo que o paciente e sua família sejam envolvidos e tranquilizados para a situação que estão vivendo (Silva, Monteiro \& Santos, 2015).

Tendo em vista o relacionamento interpessoal entre o enfermeiro e pacientes/familiares, deve ser estabelecida uma relação embasada em atenção e respeito. O profissional deve alcançar a minimização de dúvidas, medos e dificuldades dos usuários, assim como a capacitação dos mesmos para o enfrentamento de uma nova realidade, por meio de esclarecimentos sobre cuidados de saúde e também de sua situação clínica vivenciada, visando estimulá-los a participar das decisões de tratamento e cuidados (Coriolano-Marinus, Pavan, Lima \& Bettencourt, 2014; Silva, Monteiro \& Santos, 2015). Assim, quanto mais informações forem disponibilizadas, maior torna-se a participação e protagonismo dos pacientes e familiares frente a situações decisivas.

O manual preenche tal quesito quando mostra os cuidados/procedimentos a serem realizados, oportunizando aos pacientes e familiares o conhecimento das intervenções que irão ser realizadas. Logo, respaldados por um alicerce sólido constituído de informações fundamentadas em conteúdos científicos, o paciente adquire o poder de discernimento do certo e errado, com consequente incentivo de empoderamento e segurança para a execução do autocuidado. Dispor dessas tecnologias para a educação em saúde permite propiciar o aumento da autonomia, recuperação, bem-estar físico, mental e social dos usuários (Oliveira, Lucena \& Echer, 2014; Costa, Dias, Azevedo \& Silva, 2015).

No entanto, a vivência de uma situação cirúrgica impacta no paciente uma desestruturação física, biológica e emocional. Caracterizado como um momento crítico e complexo devido ao medo do desconhecido, o procedimento cirúrgico faz emergir sentimentos, como: perda de autonomia, solidão, estresse, medo, ansiedade e mudança de hábitos (Ascari et al., 2013). Enfatizase a grande valia de uma assistência de enfermagem de qualidade, com orientações que atendam e suprem todas as necessidades dos pacientes e seus familiares.

O manual poderá oferecer a promoção de bem-estar para pacientes e familiares, através da diminuição do estresse e ansiedade, do fornecimento de segurança e calma e da contribuição para a recuperação do paciente. As orientações devem esclarecer dúvidas e explicar possíveis situações que poderão ser vivenciadas. Ainda, o uso de materiais educativos durante o processo de orientação serve como instrumento de apoio aos profissionais envolvidos na assistência, permitindo que atuem como multiplicadores dos conhecimentos adquiridos, além de facilitar o entendimento e minimizar a ansiedade que muitas vezes pacientes e familiares apresentam (Silva, Monteiro \& Santos, 2015).

\section{Conclusão}

Considerando que no contexto da educação em saúde o enfermeiro é um membro essencial para a desenvoltura desse cuidado, deve empregar envolvimento, dedicação e comprometimento em tal prática. Neste sentido, foi elaborado o manual de cuidados de enfermagem para pacientes e familiares, seguindo etapas propostas na literatura, com o intuito de obter maior rigor metodológico no processo de elaboração, refletindo em melhor qualidade do material construído.

Destaca-se que de forma unânime para os participantes do estudo, as informações contidas no manual são importantes, além de que a forma de disposição das informações é adequada e as ilustrações contribuem para o melhor entendimento do texto. 
O manual é relevante, pois incentiva para a educação em saúde, mostra os cuidados e procedimentos a serem realizados e proporciona bem-estar. Tais aspectos, evidenciam o alcance dos objetivos propostos quando se faz o uso da comunicação escrita e visual através de materiais educativos. Além de que, esses podem ser utilizados como material de apoio para a execução da prática de orientações, fortalecendo o relacionamento do binômio enfermeiro-pacientes/familiares.

Portanto, é esperado que esse estudo possa contribuir acerca da conscientização de enfermeiros para iniciativas de produção de materiais educativos e para o despertar de uma assistência integral, com vistas a identificação de necessidades locais dos sujeitos e ao alcance de esferas físicas, psicológicas e espirituais. Servindo como potencializador para as ações de educação em saúde dos enfermeiros, já que a prática de educar deve ser pensada intrínseca ao cuidado de enfermagem.

Visto que esse estudo reforça a importância da prática de educação em saúde com o apoio de materiais educativos, destaca-se a necessidade de trabalhos futuros que envolvam a elaboração, avaliação e uso de materiais educativos, nas diferentes situações clínicas a depender da realidade local do pesquisador. Salienta-se a relevância de mais estudos que avaliem os reflexos para profissionais e pacientes em relação a utilização de materiais educativos por um período de tempo maior.

O manual encontra-se disponível para o público na sua versão online, no formato de ebook através do endereço eletrônico: < http://www.eenf.furg.br/documentos> e na UCC do hospital.

\section{Referências}

Ascari, R. A, Neiss, M., Sartori, A. A., Silva, O. M., Ascari, T. M., \& Galli, K. S. B. (2013). Perceptions of surgical patient during preoperative period concerning nursing care. Rev enferm UFPE on line, 21(4), 1136-44.

Benevides, L. M. B., Silva, L. F., Silva, G. C., \& Farias, M. S. (2020). Práticas educativas no controle da ansiedade de pacientes em pré-operatório de cirurgia cardíaca: revisão integrativa. Rev Fun Care Online, 12, 437-443.

Chaves, P. L., Costa, V. T., \& Lunardi, V. L. (2005). A enfermagem frente aos direitos de pacientes hospitalizados. Texto e Contexto Enfermagem, 14(1), 3843.

Coriolano-Marinus, M. W. L., Pavan, M. I, Lima, L. S., \& Bettencourt, A. R. C. (2014). Validation of educational material for hospital discharge of patients with prolonged domiciliary oxygen prescription. Esc Anna Nery Rev enferm, 18(2).

Costa, C. I. A., Pacheco, S. T. A., Soeiro, G., Adame, D. G., \& Peres, P. L. P.; Araújo, B. B. M. (2018). Construção e validação de materiais educativos para criança com doença crônica: uma revisão integrativa. Rev enferm UERJ, 26e:34208.

Costa, K. A. U., Dias, R. S., Azevedo, P. R., \& Silva, L. D. C. (2015). A importância das orientações de enfermagem no cuidado ao paciente submetido à cirurgia cardíaca: revisão integrativa. Vita et Sanitas, 9(2).

Graciotto, A., Gomes, C. J., Sangalli, E., Schneider, S., \& Echer, I. C. (2010). Sequelas Neurológicas: orientações para pacientes e familiares, 38. Hospital de Clínicas. Porto Alegre - UFRGS.

Gurusamy, K. S., Vaughan, J., \& Davidson, B. R. Formal education of patients about tounder go laparoscopic cholecystectomy. Cochrane Database of Systematic Reviews, 2, 2-34.

Hinkle, J. L., \& Cheever, K. H. (2015). Brunner e Suddarth: tratado de enfermagem médico-cirúrgica. (13a ed.), Guanabara Koogan.

Hospital Universitário. (2016). Relatório de procedimentos cirúrgicos: 2010-2015. Universidade Federal do Rio Grande (FURG).

Mafetoni, R. R., Higa, R., \& Bellini, N. R. (2011). Comunicação Enfermeiro-Paciente no pré operatório: revisão integrativa. Rev Rene, 12(4), 859-65.

Ministério da Saúde. (2016). Departamento de Informática do Sistema Único de Saúde (DATASUS). Procedimentos hospitalares do SUS por local de internação (Brasil). 〈http://tabnet.datasus.gov.br/cgi/tabcgi.exe?sih/cnv/qiuf.def>

Moraes, R., \& Gallizzi, M. do C. (2011). Análise textual discursiva. (2a ed.), Editora Unijuí.

Moura, I. H., Silva, A. F. R., Rocha, A. E. S. H., Lima, L. H. O., Moreira, T. M. M. \& Silva, A. R. V. (2017). Construção e validação de material educativo para prevenção de síndrome metabólica em adolescentes. Rev Latino-Am. Enfermagem, 25e:2934.

Nascimento, E. A., Tarcia, R. M. L., Magalhães, L. P., Soares, M. A. L., Suriano, M. L. F., \& Domenico, E. B. L. (2015). Educational pamphlets on health: a reception study. Rev Esc Enferm USP. 49(3).

Oliveira, M. C., Lucena, A. F., \& Echer, I. C. (2014). Sequelas Neurológicas: elaboração de um manual de orientação para o cuidado em saúde. Rev enferm UFPE on line, 8(6)1597-603. 
Research, Society and Development, v. 10, n. 11, e205101119521, 2021

(CC BY 4.0) | ISSN 2525-3409 | DOI: http://dx.doi.org/10.33448/rsd-v10i11.19521

Oliveira, S. C., Lopes, M. V. O.; \& Fernandes A. F. C. (2014). Development and validation of an educational booklet for healthy eating during pregnancy. Rev latinoam enferm, 22(4).

Rigon, A. G., \& Neves, E. T. (2011). Educação em saúde e a atuação de enfermagem no contexto de unidades de internação hospitalar: o que tem sido ou há para ser dito? Texto e Contexto Enfermagem, 20(4), 812-817.

Silva, R. C. A., Monteiro, G. L., \& Santos, A. G. O. (2015). Enfermeiro na Educação de Cuidadores de pacientes com sequelas de Acidente Vascular Cerebral. Revista de Atenção à Saúde, 13(45), 114-120.

Stinton, L. M., \& Shaffer, E. A. (2012). Epidemiology of Gallbladder Disease: Cholelithiasis and Cancer. Gut and Liver, 6(2). 